\title{
Regulatory Network of MicroRNAs, Target Genes, Transcription Factors and Host Genes in Endometrial Cancer
}

\author{
Lu-Chen $X^{1,3}$, Zhi-Wen $\mathrm{Xu}^{2,3 *}$, Kun-Hao Wang ${ }^{2,3}$, Ning Wang ${ }^{2,3}$, Xiao-Xu \\ Zhang ${ }^{1}$, Shang Wang ${ }^{2,3}$
}

\begin{abstract}
Genes and microRNAs (miRNAs) have important roles in human oncology. However, most of the biological factors are reported in disperse form which makes it hard to discover the pathology. In this study, genes and miRNAs involved in human endometrial cancer(EC) were collected and formed into regulatory networks following their interactive relations, including miRNAs targeting genes, transcription factors (TFs) regulating miRNAs and miRNAs included in their host genes. Networks are constructed hierarchically at three levels: differentially expressed, related and global. Among the three, the differentially expressed network is the most important and fundamental network that contains the key genes and miRNAs in EC. The target genes, TFs and miRNAs are differentially expressed in EC so that any mutation in them may impact on EC development. Some key pathways in networks were highlighted to analyze how they interactively influence other factors and carcinogenesis. Upstream and downstream pathways of the differentially expressed genes and miRNAs were compared and analyzed. The purpose of this study was to partially reveal the deep regulatory mechanisms in EC using a new method that combines comprehensive genes and miRNAs together with their relationships. It may contribute to cancer prevention and gene therapy of EC.
\end{abstract}

Keywords: Regulatory network - endometrial cancer - differentially expressed gene - microRNA - transcription factor

Asian Pac J Cancer Prev, 16 (2), 475-483

\section{Introduction}

$\mathrm{EC}$ is the fourth most common malignancy in women in the developed world after breast, colorectal and lung cancer. Worldwide, similar to 74,000 women die from endometrial cancer each year (Bell, 2014). Molecular targeted therapies are now a focus of attention. They may provide useful future strategies for control of endometrial malignancies in developing countries and across the world (Thanapprapasr et al., 2013). However, the molecular events involved in the development and progression of EC remain unclear ( $\mathrm{Li}$ et al., 2013). In this study, we mainly focus on the relationships between genes and miRNAs in $\mathrm{EC}$ in order to discover the regulation mechanism.

TFs and miRNAs are prominent regulators for gene expression (Hobert, 2008). TFs are proteins that can activate or repress transcription by binding to the upstream regions of genes (Tran et al., 2010).

MiRNAs comprise a family of small, non-coding RNAs, which regulate gene expression at the posttranscriptional level (Torres et al., 2013). In EC, miRNAs are associated with regulation of gene expression, epigenetic dysfunction and carcinogenesis, thus, miRNAs are likely to have key roles in diagnosis, prognostic prediction, and therapy in EC (Banno et al., 2013).
The relations between miRNAs and target genes are studied. MiRNAs regulate a variety of cellular processes via the regulation of multiple target genes. They control the cellular expression machinery and have a profound impact on the regulation of signaling pathways (Lv et al., 2012).

Host genes are genes where certain miRNAs locate. It is indicated that miRNAs are transcribed in parallel with their host transcripts and two different transcription classes of miRNAs, exonic and intronic, were identified (Rodriguez et al., 2004). Intronic miRNA and its host gene have a close relation (Baskerville et al., 2005).

Numerous experimental data suggest that the differentially expressed genes and miRNAs contribute to the pathogenesis of EC, while related genes and miRNAs also play a part. However, the data can be found in scattered form, which makes it difficult to analyze the mechanism of EC systematically. In this study, genes and miRNAs are used to construct three networks including differentially expressed network, related network and global network. Each network is combined with three relationships of miRNAs targeting at genes, TFs regulating miRNAs and miRNAs locating on their host genes. Then contrast tables about upstream and downstream of genes and miRNAs are extracted to find the similarities and figure out differences. Crucial genes and miRNAs in the 
networks are focused on to reveal the pathogeneses of EC. Our teammates have successfully analyzed retinoblastoma using the same method (Li et al., 2013). This study provides a new point of view to investigate EC.

\section{Materials and Methods}

Data collection and processing of genes and miRNAs

The comprehensive data of EC were manually collected from databases and literatures. Symbols of genes were unified officially by checking them in NCBI database (http://www.ncbi.nlm.nih.gov/gene).

The experimentally validated dataset of human miRNAs and their target genes was collected from the Tarbase (http://diana.cslab.ece.ntua.gr/tarbase/) (Papadopoulos et al., 2009), miRTarBase (http:// mirtarbase.mbc.nctu.edu.tw/) (Hsu et al., 2014) and miRecords (http://mirecords.biolead.org/) (Xiao et al., 2009). The dataset was considered as set $U_{1}$.

The dataset of human TFs regulating miRNAs was collected from TransmiR (http://mirtarbase.mbc.nctu. edu.tw/)(Wang et al., 2010) and was considered as set $U_{2}$.

The host genes of human miRNAs were extracted from miRBase (http://www.mirbase.org/) (Kozomara et al.,2011) and NCBI. The dataset was considered as set $U_{3}$.

Differentially expressed genes of EC were collected from NCBI SNP database (http://www.ncbi.nlm.nih. gov/snp/), KEGG (www.genome.jp/kegg/pathway. html), Cancer GeneticsWeb (http://www.cancerindex. org/geneweb/clink30.htm) and pertinent literatures. All the dataset of the differentially expressed genes was considered as set $U_{4}$.

Related genes were collected from GeneCards database (http://www.genecards.org/) and pertinent literatures. In GeneCards database, the first 100 relative genes were picked out. Furthermore, some TFs predicted by Pmatch method (Chekmenev et al., 2005) were also included in related genes. 1000nt promoter region sequences of differentially expressed genes' targets were downloaded from UCSC database (http://genome.ucsc.edu/) (Fujita et al., 2011). Pmatch method that combines pattern matching and weight matrix approaches was used to identify transcription factor binding sites (TFBSs) in 1000nt promoter region sequences and mapped TFBSs onto promoter region of targets. Matrix library of Pmatch is also sets of known TF-binding sites collected in TRANSFAC, so it provides the possibility to search for a large variety of different TF binding sites. The vertebrate matrix was used and be restricted with high quality criterion. The dataset of the related genes was considered as set $U_{5}$.

Differentially expressed miRNAs were collected from mir2Disease (http://www.mir2disease.org/) (Jiang et al., 2009) and pertinent literatures. Related miRNAs were extracted from pertinent literatures. The datasets of differentially expressed miRNAs and related miRNAs were separately considered as set $U_{6}$ and set $U_{7}$.

\section{Three networks construction}

Three networks of EC were constructed, which are differentially expressed network, related network and global network. Set $U_{1}, U_{2}$ and $U_{3}$ were combined to get the global network. The global network includes all the regulatory relations of miRNAs targeting at targets, TFs regulating miRNAs and host genes including miRNAs. It is a reference to get the differentially expressed network and related network.

The relations between differentially expressed genes and differentially expressed miRNAs that included in the global network were selected to constitute the differentially expressed network. If a pair of gene and miRNA extracted from $U_{4}$ and $U_{6}$ can be found in the global network, the elements and the relation can be included in the differentially expressed network. The related network was constructed by using the same method with elements in set $U_{5}$ and $U_{7}$ finally, the software Cytoscape was used to show the network graphically. It helps to analyze the regulatory pathways in EC.

\section{Results}

\section{Differentially expressed miRNAs in EC}

MiRNAs are emerging as important regulators in cancer cells. Accumulating evidence has demonstrated that miRNAs play critical roles in cancer initiation and development by functioning either as oncogenes or as tumor suppressors (Ye et al., 2014). Aberrant expression of miRNAs including miR-200b, miR-130a/b, miR-625 and miR-222 was associated with tumorigenesis and metastasis in EC (Li et al., 2013). MiR-185, miR-106a, miR-181a, miR-210, miR-423, miR-103, miR-107, miRlet7c, miR-205, miR-449, and miR-429 show enhanced expression in EC tissues compared with normal tissues, whereas miR-let7e, miR-221, miR-30c, miR-152, miR193, miR-204 miR-99b, and miR-193b have decreased expression in cancer tissues (Banno et al., 2013). MiR155 is one of the miRNAs most consistently involved in neoplastic diseases and it is overexpressed in EC tissues (Choi et al., 2012). Quantitative realtime polymerase chain reactions were performed to validate the results that the expression levels of miR-625*, miR-21, miR-142-5p and miR-146b-5p were increased, whereas the expression levels of miR-633, miR-29c and miR-29* were decreased (Bae et al., 2012). MiR-125b was downregulated in $\mathrm{EC}$ and regulated proliferative, metastatic and invasive activities (Shang et al., 2012; Banno et al., 2013). In recent studies, miRNAs were found up-regulated in EC including miR-200 family, miR-9 and miR-203, whereas miRNAs including miR-410, miR-214, miR-99a, miR-199b, miR100 , and miR-424 were found down-regulated (Torres et al.,2013). Reduced expression of miR-199a-3p was found in human endometrioid endometrial cancer specimens, which could occur in various malignant tumors and was closely linked with tumor cell proliferation, invasion and metastasis (Wu et al., 2013). MiR-503 acts as tumor-suppressor in endometrioid endometrial cancer, and abnormal suppression of miR-503 leads to an increase in the CCND1 level, which may promote carcinogenesis and progression (Wu et al., 2013). Compared with the miRNAs in the normal endometrium, miR-15b, miR17-5p, miR-20a, miR-125a, miR-221 and miR-222 were significantly down-regulated and miR-200b was significantly up-regulated in the cancerous endometrium 
(Ramon et al., 2012).

\section{Differentially expressed genes in EC}

It is generally accepted that the alterations in the expression of critical genes take major responsibility for the evolution of cancer. CDH1, SDHB, MLH3 and MSH2 can be found in the NCBI SNP database. KRAS, CTNNB1 and ERBB2 can be found in KEGG database. PTEN, TP53, SLC2A1, MSH6, MSH3 and RBL2 come from the Cancer GeneticsWeb database. In pertinent literatures, mutations in PIK3CA, KRAS and PTEN were reported (Machay et al., 2014). In a research of 243 endometrial tumors, PTEN was the most frequently mutated gene (108 tumors, 44\%) followed by PIK3CA (97 tumors, 40\%) (Cheung, 2012). Moreover, FGFR2, CTNNB1, TP53, KRAS and AKT1 mutations in samples were revealed (Cheung, 2012). Mutations in ESCO1, CHTF18 and MRE11A were uncovered in $\mathrm{EC}$, and the cooccurrence of mutations in ESCO1 and CHTF18 was statistically significant (Price et al., 2013). Mutations in MSH6, TGFBR2 and BAX were found frequently in EC (Kawaguchi et al., 2009). Twist homolog 1 (TWIST1) is a transcription factor and serves as a powerful oncogene, which has been observed to be up-regulated expression and associated with metastasis in several cancers including EC (Li et al., 2014). COPZ2 in EC was frequently down-regulated (Tsuruta et al., 2011). FOXO1, a crucial regulator of progesterone-dependent differentiation of the normal human endometrium, could be repressed expression by several miRNAs (Xu et al., 2013). CCND1 was up-regulated in EC targeted by the down-regulated potential tumor suppressor miR-503(Xu et al., 2013). DICER1 was higher in normal endometrium than in EC (Li et al., 2013).

\section{Differentially expressed network of EC}

There are 5 TFs (PTEN, TP53, TWIST1, CCND1 and AKT1), another 17 differentially expressed genes, 49 differentially expressed miRNAs and 56 host genes in differentially expressed network. It is composed of three relationships including TFs regulating miRNAs, miRNAs targeting at target genes and host genes including miRNAs. The single nodes that do not have regulatory relations with others are omitted in Figure 1 though they play a function too. The differentially expressed network contains many incorrect data linkages when EC emerges, so it can be used as a faulty map of EC. According to the faulty map, we can systematically get the knowledge of the regulation relations in $\mathrm{EC}$.

The TFs are regarded as a kind of special genes. The 5 TFs related pathways are most significant in Figure 1. TP53 and PTEN play key roles in EC. TP53 and PTEN mutations occur in EC frequently and the mutations of two genes could occur at the same time, suggesting that both these genes participate in the development of EC (Janiec-Jankowska et al., 2010). In differentially expressed network, 3 miRNAs(hsa-miR-221, hsa-miR-222 and hsamiR-125b) target at TP53 which regulates 6 miRNAs (hsamiR-200b, hsa-miR-200c, hsa-miR-125b, hsa-miR-155, hsa-miR-107 and hsa-miR-29c). 6 miRNAs(Hsa-miR-21, hsa-miR-214, hsa-miR-20a, hsa-miR-17-5p, hsa-miR-221 and hsa-miR-222) target at PTEN which regulates hsamiR-21. 7 miRNAs (hsa-miR-155, -20a, -15b, -503, $-193 \mathrm{~b},-424,-449 a)$ target at CCND1 which regulates hsa-miR-20a. TWIST1, which serves as a powerful

Table 1. The Upstream and Downstream of PTEN in three Networks of EC

\begin{tabular}{|c|c|c|c|c|c|c|}
\hline \multicolumn{3}{|c|}{ Upstream (miRNAs that target gene) } & \multirow[t]{2}{*}{ Gene } & \multicolumn{3}{|c|}{ Downstream (miRNA that is regulated by gene) } \\
\hline $\begin{array}{l}\text { Differentially } \\
\text { expressed network }\end{array}$ & Related network & $\overline{\text { Global network }}$ & & $\begin{array}{c}\text { Differentially } \\
\text { expressed network }\end{array}$ & Related network & Global network \\
\hline $\begin{array}{l}\text { hsa-miR-17-5p } \\
\text { hsa-miR-20a } \\
\text { hsa-miR-21 } \\
\text { hsa-miR-214 } \\
\text { hsa-miR-221 } \\
\text { hsa-miR-222 }\end{array}$ & $\begin{array}{l}\text { hsa-miR-17-5p } \\
\text { hsa-miR-20a } \\
\text { hsa-miR-21 } \\
\text { hsa-miR-214 } \\
\text { hsa-miR-221 } \\
\text { hsa-miR-222 } \\
\text { hsa-miR-106b } \\
\text { hsa-miR-18a } \\
\text { hsa-miR-26a }\end{array}$ & $\begin{array}{l}\text { hsa-miR-106b } \\
\text { hsa-miR-141 } \\
\text { hsa-miR-17 } \\
\text { hsa-miR-17-5p } \\
\text { hsa-miR-18a } \\
\text { hsa-miR-19a } \\
\text { hsa-miR-19b } \\
\text { hsa-miR-20a } \\
\text { hsa-miR-21 } \\
\text { hsa-miR-214 } \\
\text { hsa-miR-21-5p } \\
\text { hsa-miR-216a } \\
\text { hsa-miR-217 } \\
\text { hsa-miR-22 } \\
\text { hsa-miR-221 } \\
\text { hsa-miR-221-3p } \\
\text { hsa-miR-222 } \\
\text { hsa-miR-222-3p } \\
\text { hsa-miR-26a } \\
\text { hsa-miR-29a } \\
\text { hsa-miR-29b-3p } \\
\text { hsa-miR-494 } \\
\text { hsa-miR-519a-3p } \\
\text { hsa-miR-519d } \\
\text { hsa-miR-93-5p }\end{array}$ & PTEN & hsa-miR-21 & $\begin{array}{c}\text { hsa-miR-21 } \\
\text { hsa-miR-302 }\end{array}$ & $\begin{array}{l}\text { hsa-miR-19a } \\
\text { hsa-miR-21 } \\
\text { hsa-miR-22 } \\
\text { hsa-miR-25 } \\
\text { hsa-miR-302 } \\
\text { hsa-miR-302a } \\
\text { hsa-miR-302b } \\
\text { hsa-miR-302c } \\
\text { hsa-miR-302d } \\
\text { hsa-miR-302f }\end{array}$ \\
\hline
\end{tabular}


Lu-Chen Xue et al

Table 2. The Upstream and Downstream of hsa-miR-21 in Three Networks of EC

\begin{tabular}{|c|c|c|c|c|c|}
\hline $\begin{array}{l}\text { Differentially } \\
\text { expressed network }\end{array}$ & $\begin{array}{c}\text { Upstream } \\
\text { Related network }\end{array}$ & Global network & $\begin{array}{ll}\text { miRNAs } & \begin{array}{c}\text { Differentially } \\
\text { expressed network }\end{array}\end{array}$ & $\begin{array}{c}\text { Downstream } \\
\text { Related network }\end{array}$ & Global network \\
\hline 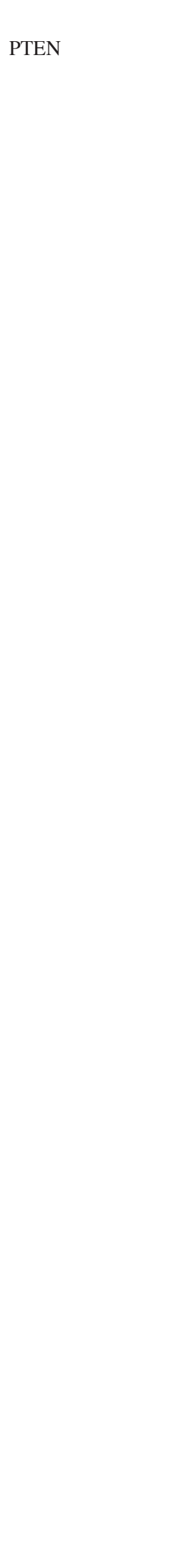 & $\begin{array}{l}\text { PTEN } \\
\text { EGFR } \\
\text { NFKB1 } \\
\text { REL } \\
\text { RELA }\end{array}$ & $\begin{array}{l}\text { BMP6 } \\
\text { BMPR1A } \\
\text { BMPR1B } \\
\text { DDX5 } \\
\text { EGFR } \\
\text { ESR1 } \\
\text { ETV5 } \\
\text { FOXO3 } \\
\text { JUN } \\
\text { NFIB } \\
\text { NFKB1 } \\
\text { PTEN } \\
\text { RASGRF1 } \\
\text { REL } \\
\text { RELA } \\
\text { REST } \\
\text { STAT3 } \\
\text { STAT4 } \\
\text { TCF4 }\end{array}$ & 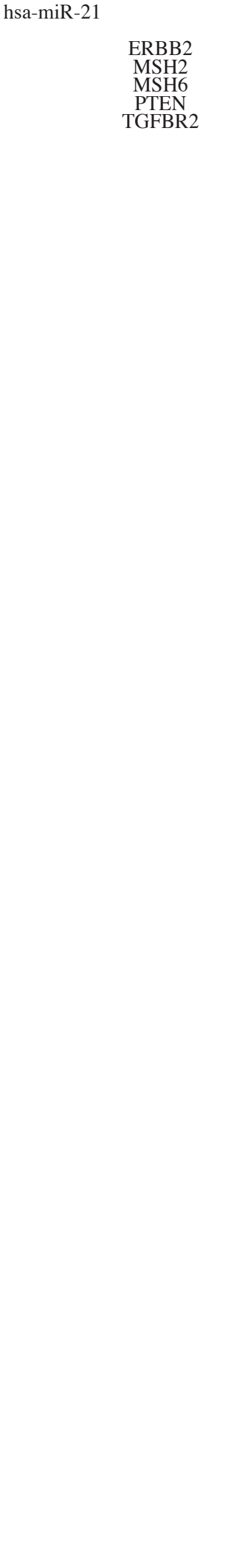 & $\begin{array}{c}\text { ERBB2 } \\
\text { MSH2 } \\
\text { MSH6 } \\
\text { PTEN } \\
\text { TGFBR2 } \\
\text { CDC25A } \\
\text { CDKN1A } \\
\text { E2F1 } \\
\text { E2F2 } \\
\text { EGFR } \\
\text { NCOA3 } \\
\text { PDCD4 } \\
\text { SERPINB5 } \\
\text { TGIF1 } \\
\text { TIMP3 }\end{array}$ & $\begin{array}{l}\text { ACTA2 } \\
\text { ANKRD46 } \\
\text { APAF1 } \\
\text { BASP1 } \\
\text { BCL2 } \\
\text { BMPR2 } \\
\text { BTG2 } \\
\text { CCR1 } \\
\text { CDC25A } \\
\text { CDK2AP1 } \\
\text { CDK6 } \\
\text { CDKN1A } \\
\text { CFL2 } \\
\text { DAXX } \\
\text { DERL1 } \\
\text { E2F1 } \\
\text { E2F2 } \\
\text { EGFR } \\
\text { EIF2S1 } \\
\text { EIF4A2 } \\
\text { ERBB2 } \\
\text { FAM3C } \\
\text { FAS } \\
\text { FMOD } \\
\text { GLCCI1 } \\
\text { HIPK3 } \\
\text { HNRNPK } \\
\text { ICAM1 } \\
\text { IL1B } \\
\text { IL6R } \\
\text { ISCU } \\
\text { JAG1 } \\
\text { JMY } \\
\text { LRRFIP1 } \\
\text { MARCKS } \\
\text { MEF2C } \\
\text { MSH2 } \\
\text { MSH6 } \\
\text { MTAP } \\
\text { MYC } \\
\text { NCAPG } \\
\text { NCOA3 } \\
\text { NFIB } \\
\text { NT-3 } \\
\text { PCBP1 } \\
\text { PDCD4 } \\
\text { PDHA2 } \\
\text { PELI1 } \\
\text { PLAT } \\
\text { PLOD3 } \\
\text { PPARA } \\
\text { PPIF } \\
\text { PRRG4 } \\
\text { PTEN } \\
\text { PTX3 } \\
\text { RASA1 } \\
\text { RASGRP1 } \\
\text { RECK } \\
\text { REST } \\
\text { RHOB } \\
\text { RP2 } \\
\text { RPS7 } \\
\text { RTN4 } \\
\text { SERPINB5 } \\
\text { SESN1 } \\
\text { SGK3 } \\
\text { SLC16A10 } \\
\text { SOCS5 } \\
\text { SOX5 } \\
\text { SPATS2L } \\
\text { SPRY2 } \\
\text { STAT3 } \\
\text { TGFBI } \\
\text { TGFBR2 } \\
\text { TGFBR33 } \\
\text { TGIF1 } \\
\text { TIAM1 } \\
\text { TIMP3 } \\
\text { TM9SF3 } \\
\text { TNFAIP3 } \\
\text { TOPORS } \\
\text { TP53BP2 } \\
\text { TP63 } \\
\text { TPM1 } \\
\text { WFS1 } \\
\text { WIBG }\end{array}$ \\
\hline
\end{tabular}


oncogene (Li et al., 2014), can regulate hsa-miR-214, hsamiR-205, hsa-miR-200b and hsa-miR-200c. The present study implicates TWIST1 as a potential therapeutic target for this tumor type. The regulatory relations about TFs indicate that TFs are important biological factors in EC. The miRNAs that regulated by TFs can also influence their targets. The targets of miRNAs such as RBL2, DNMT3B, PIK3R1, CTNNB1, TGFBR2, MLH1, KRAS, MSH6, MSH2, DICER1, ERBB2 and FBXW7 do not regulate any miRNA. These genes can probably influence the tumorigenesis of EC. For example, KRAS is a typical oncogene in EC (Krakstad et al., 2012). ERBB2 encodes a member of the epidermal growth factor receptor family of receptor tyrosine kinases, and amplification and/or overexpression of ERBB2 has been reported in other numerous cancers, including head and neck cancer, gastric cancer and ovarian cancers (Shang et al., 2012). We also find something interesting in the differentially expressed network. TWIST1 and TP53 could co-regulate hsa-miR200b and hsa-miR-200c. AKT1 and TP53 co-regulate hsa-miR-155, hsa-miR-125b-1 and hsa-miR-125b-2. PTEN and CCND1 are both targeted by hsa-miR-20a. PTEN and TP53 are both targeted by hsa-miR-221 and hsa-miR-222. AKT1 and TP53 are both targeted by hsamiR-125b-1 and hsa-miR-125b-2. PTEN and hsa-miR-21 form feedback-loops (FBLs), that is PTEN regulates hsamiR-21 and hsa-miR-21 targets at PTEN in return. HsamiR-21 overexpression contributes to cell proliferation by targeting PTEN in EC (Qin et al., 2012). Similarly, CCND1 and hsa-miR-20a,AKT1 and hsa-miR-125b (hsamiR-125b-1, hsa-miR-125b-2), TP53 and hsa-miR-125b (hsa-miR-125b-1, hsa-miR-125b-2) form FBLs. How to balance the FBLs in EC helps much to the cancer therapy.

Besides TFs, other pathways are significant too. CDH1 is targeted by hsa-miR-9(hsa-miR-9-1, hsa-miR-9-2 and hsa-miR-9-3). Besides CDH1, hsa-miR-9 also targets at FOXO1, a crucial regulator of progesterone-dependent differentiation of the normal human endometrium which could be repressed expression by miRNA (Xu et al., 2013). Expression of miR-9 could significantly reduce the abundance of FOXO1 (Myatt et al., 2010). It shows that hsa-miR-9 is an important miRNA involved in EC. MSH2 and MSH6 are targeted by hsa-miR-155 and hsamiR-21 in common, which indicates that the two genes may have some similarities.

The functions of miRNAs that involved in differentially expressed network are worth concern. For example, hsamiR-125b can be regulated by AKT1 and TP53 and can target at DICER1, ERBB2, AKT1 and TP53. It is reported that hsa-miR-125b contributes to cells invasion partly through directly down-regulating ERBB2 expression (Shang et al., 2012). Hsa-miR-200c is widely investigated in recent years. It is significantly increased in EC regulated by TP53 and TWIST1 (Cohn et al., 2010). CCND1 has a binding sequence of miR-503 within its 3 untranslated region, and was confirmed to be a direct target of miR503 by the fluorescent reporter assays (Xu et al., 2013). Abnormal suppression of miR-503 leads to an increase in the CCND1 level, which may promote carcinogenesis and progression (Xu et al., 2013). The content changes of miRNAs help us to know how to adjust them in the pathologic network to control the occurrence of cancer. For example, hsa-miR-155 is validated to be overexpressed in the regulatory network and take effect in the pathogenesis (Choi et al., 2012). The up-regulation impacts the expression of MSH2, MSH6, MLH1, CCND1, CTNNB1 and KRAS. Correcting individual expression level not only can reduce the morbidity of EC, but also rectify the genes and miRNAs downstream to normal standard.

The relation of host genes and its miRNA presents some special features in differentially expressed network. The mutation of host genes may lead to the differentially expression of miRNAs that included in it. Every differentially expressed miRNA in Figure 1 has host gene. A host gene may include one more miRNAs while a miRNA may come from more than one host genes. Hsa-miR-9-1, hsa-miR-9-2 and hsa-miR-9-3 have their own host genes and hsa-miR-9-2 locates in two host genes, MIR9-2 and LINC00461. Hsa-let-7c has three host genes including LINC00478 while LINC00478 hosts three miRNAs including hsa-let-7c, hsa-miR-99a and hsa-miR-125b-2. The host genes are not differentially expressed in EC except COPZ2, which is the host gene of hsa-miR-152. Epigenetic silencing documented in miR152 was consistent with its location at $17 \mathrm{q} 21.32$ in intron 1 of the COPZ2 gene (Tsuruta et al., 2011). It is noted that the frequencies of down-regulation of COPZ2 in EC cell lines and primary cases $(<0.5$-fold expression) were $100 \%$ (13 of 13) and $75.7 \%$ (53 of 70) (Tsuruta et al., 2011). The method of researching differentially expressed miRNAs' host genes helps to research the pathogenesis of cancers.

We can conclude that investigating the differentially expressed genes and miRNAs has great significance in cancer prevention and gene therapy. The differentially expressed network partially reveals the mechanism of EC. If the faulty regulations can be corrected, EC may be prevented and even cured in theory.

\section{Related network of EC}

Related network of EC is more complicated than the differentially expressed network because it contains more elements and pathways. Besides differentially expressed genes and miRNAs, the related genes and miRNAs are also involved in EC-related network. New added related genes not only regulate differentially expressed miRNAs but also regulate related miRNAs. New added related miRNAs can target at differentially expressed genes and related genes. For example, hsa-miR-152 is a differentially expressed miRNA, which targets at DNMT1 in differentially expressed network. In related network it targets at added related genes E2F3, MET and MTOR (Banno et al., 2013). The differentially expressed gene TWIST1 has an added miRNA hsa-miR-106b that targets at it in related network. It is suggested that hsa-miR-106b could suppress EC cell invasion by down-regulating TWIST1 expression (Dong et al., 2014). CDC25A, a new added related gene, could be targeted by 5 miRNAs (hsa-miR-21, hsa-miR-424, hsa-miR-449a, hsa-miR-503 and hsa-miR-125b) that are all differentially expressed miRNAs. Hsa-miR-449a may act as a tumor suppressor by targeting CDC25A in EC (Ye et al., 2014). The other 
Table 3. The Upstream and Downstream of E2F1 in three Networks of EC

\begin{tabular}{|c|c|c|c|c|c|c|}
\hline \multicolumn{3}{|c|}{ Upstream (miRNAs that target gene) } & \multirow[b]{2}{*}{$\mathrm{TF}$} & \multicolumn{3}{|c|}{ Downstream (miRNA that is regulated by gene) } \\
\hline $\begin{array}{l}\text { Differentially } \\
\text { expressed network }\end{array}$ & Related network & Global network & & $\begin{array}{c}\text { Differentially } \\
\text { expressed network }\end{array}$ & Related network & Global network \\
\hline $\begin{array}{l}\text { hsa-miR-106a } \\
\text { hsa-miR-17-5p } \\
\text { hsa-miR-20a } \\
\text { hsa-miR-21 }\end{array}$ & $\begin{array}{l}\text { hsa-miR-106a } \\
\text { hsa-miR-106b } \\
\text { hsa-miR-17-5p } \\
\text { hsa-miR-20a } \\
\text { hsa-miR-21 }\end{array}$ & $\begin{array}{l}\text { hsa-let-7a } \\
\text { hsa-miR-106a } \\
\text { hsa-miR-106a-5p } \\
\text { hsa-miR-106b } \\
\text { hsa-miR-126 } \\
\text { hsa-miR-149* } \\
\text { hsa-miR-17 } \\
\text { hsa-miR-17-5p } \\
\text { hsa-miR-203a } \\
\text { hsa-miR-20a } \\
\text { hsa-miR-21 } \\
\text { hsa-miR-223 } \\
\text { hsa-miR-23b } \\
\text { hsa-miR-330-3p } \\
\text { hsa-miR-331-3p } \\
\text { hsa-miR-34a } \\
\text { hsa-miR-93 } \\
\text { hsa-miR-98 }\end{array}$ & E2F1 & $\begin{array}{l}\text { hsa-miR-106a } \\
\text { hsa-miR-15b } \\
\text { hsa-miR-20a } \\
\text { hsa-miR-449a } \\
\text { hsa-miR-449b }\end{array}$ & $\begin{array}{c}\text { hsa-miR-106a } \\
\text { hsa-miR-15b } \\
\text { hsa-miR-20a } \\
\text { hsa-miR-449a } \\
\text { hsa-miR-449b } \\
\text { hsa-miR-106b } \\
\text { hsa-miR-18a }\end{array}$ & $\begin{array}{l}\text { hsa-let-7a } \\
\text { hsa-let-7a-1 } \\
\text { hsa-let-7a-2 } \\
\text { hsa-let-7a-3 } \\
\text { hsa-let-7i } \\
\text { hsa-miR-106 } \\
\text { hsa-miR-106a } \\
\text { hsa-miR-106b } \\
\text { hsa-miR-15a } \\
\text { hsa-miR-15b } \\
\text { hsa-miR-16 } \\
\text { hsa-miR-16-1 } \\
\text { hsa-miR-16-2 } \\
\text { hsa-miR-16-3 } \\
\text { hsa-miR-17 } \\
\text { hsa-miR-18a } \\
\text { hsa-miR-18b } \\
\text { hsa-miR-195 } \\
\text { hsa-miR-19a } \\
\text { hsa-miR-19b } \\
\text { hsa-miR-19b-1 } \\
\text { hsa-miR-19b-2 } \\
\text { hsa-miR-20 } \\
\text { hsa-miR-20a } \\
\text { hsa-miR-20b } \\
\text { hsa-miR-223 } \\
\text { hsa-miR-25 } \\
\text { hsa-miR-363 } \\
\text { hsa-miR-449 } \\
\text { hsa-miR-449a } \\
\text { hsa-miR-449b } \\
\text { hsa-miR-449c } \\
\text { hsa-miR-91 } \\
\text { hsa-miR-92-1 } \\
\text { hsa-miR-92-2 } \\
\text { hsa-miR-92a } \\
\text { hsa-miR-92a-1 } \\
\text { hsa-miR-92a-2 } \\
\text { hsa-miR-93 }\end{array}$ \\
\hline
\end{tabular}

miRNAs may have the similar functions to CDC25A. The related network contributes to the understanding of EC additionally.

\section{Global network}

The differentially expressed network and the related network are both involved in the global network. The global network contains all the regulatory relations between all the genes and miRNAs. It is an experimentally validated biological network in human body. It can be a reference for the study of the differentially expressed network and related network, especially the first one.

Network of predicted TFs and differentially expressed miRNAs in EC

We got predicted TFs by using P-match method above. They are probably involved in transcription process of EC because they may cause the similar results with differentially expressed TFs in EC. Here the regulatory network of predicted TFs and differentially expressed
miRNAs in EC is analyzed in Figure 2. Obviously, 4 predicted TFs including E2F1, E2F3, NFKB1 and ZEB1 are more significant than others in Figure 2. E2F1 could regulate 5 miRNAs and be targeted by 4 miRNAs. NFKB 1 could regulate 8 miRNAs and be targeted by 3 miRNAs. ZEB1 regulates 5 miRNAs and is targeted by 4 miRNAs. Specifically, 8 pairs of TF and miRNA form FBLs, which are E2F1 and hsa-miR-106a, E2F1 and hsa-miR-20a, NFKB1 and hsa-miR-9-1, hsa-miR-9-2 and hsa-miR-9-3, ZEB1 and hsa-miR-429, hsa-miR-200b and hsa-miR-200c. In Figure 2, it can be seen that a TF can indirectly influence another TF through regulating some differentially expressed miRNAs. The network of predicted TFs and miRNAs indicates the possible transcription regulation in EC. It may further reveal transcription mechanism of EC.

Feature of pathways about differentially expressed genes in $E C$

To describe the regulatory relations more clearly, the 

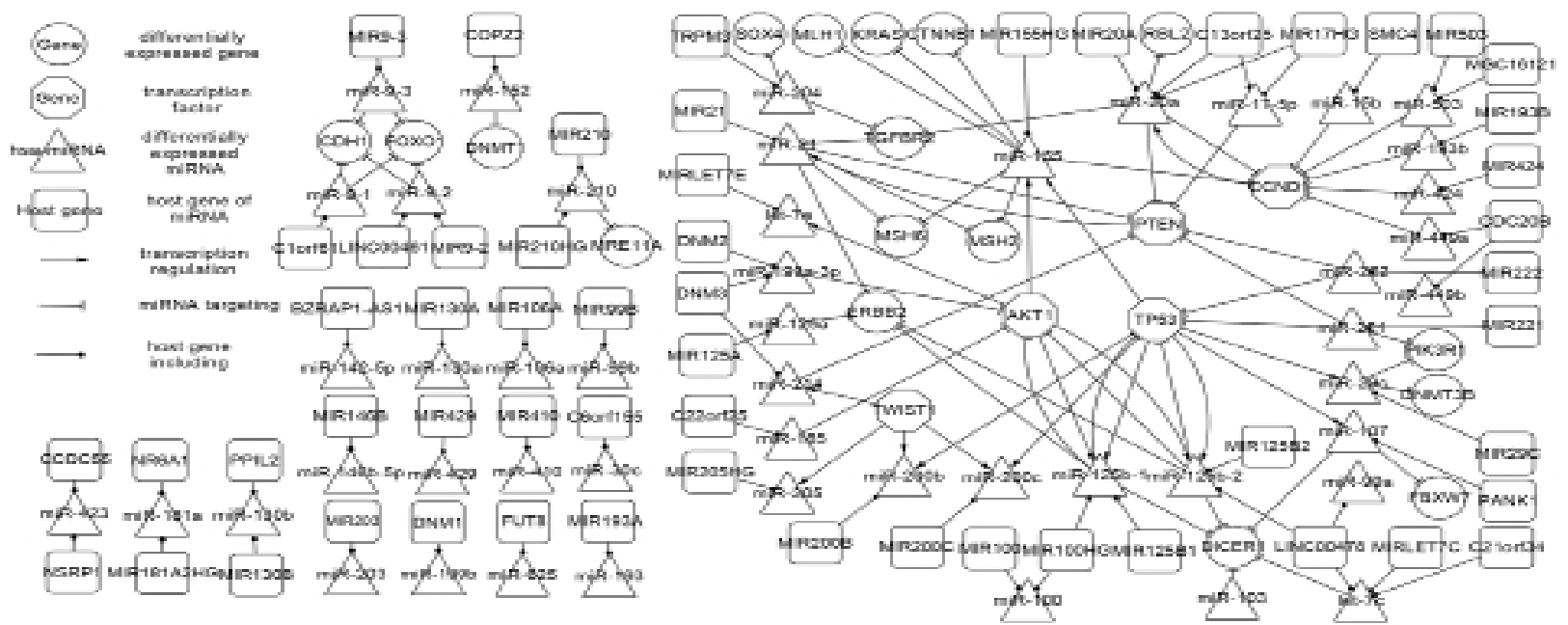

Figure 1. The Differentially Expressed Network of Genes and miRNAs in EC

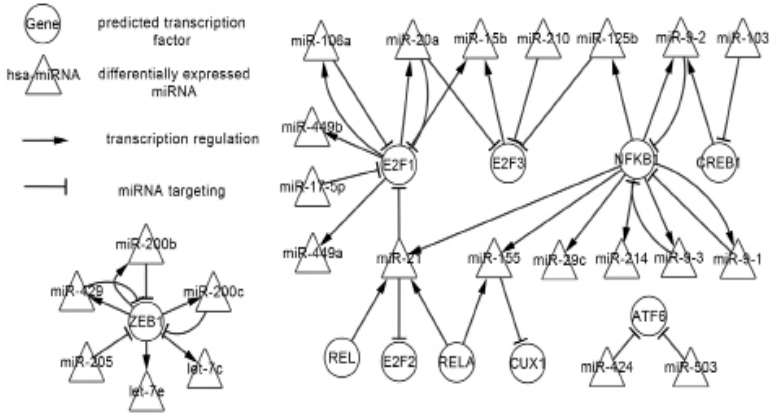

Figure 2. Network of Predicted TFs and Differentially Expressed miRNAs

upstream and downstream of differentially expressed genes, miRNAs and predicted TFs were extracted to analyze the features. Firstly we focus on the differentially expressed genes. Adjacent nodes of differentially expressed genes in three networks (differentially expressed network, related network and global network) were extracted and compared in comparison table. Upstream (miRNAs that target at genes) and downstream (miRNAs that are regulated by genes) were listed in three levels. Here we take PTEN for an example.

Table 1 shows the upstream and downstream of PTEN. PTEN is a fatal factor in EC. PTEN loss, which can be caused by gene mutation, promoter methylation, and protein degradation, was observed in 46 of 85 samples analyzed (Mackay et al., 2014). Evaluation of expression of PTEN gene may turn out to be a very useful tool for searching new diagnostic and therapeutic methods (Samulak et al., 2014).

PTEN has abundant adjacent nodes in networks of EC. There are 6 miRNAs target at PTEN which regulates 1 miRNA in differentially expressed network. Hsa-miR-21 and PTEN form FBLs. 9 miRNAs target at PTEN and PTEN regulates 2 miRNAs in related network. In global network, 25 miRNAs target at PTEN which regulates 10 miRNAs. The upstream and downstream in three networks show a terraced variation. The miRNAs in the global network provide support for EC's study. The upstream of PTEN influences the downstream indirectly by affecting on PTEN. Furthermore, the upstream and downstream can be affected by their predecessors and successors.
Feature of pathways about differentially expressed miRNAs in EC

Similarly, adjacent nodes of each differentially expressed miRNA were extracted and compared in three networks. Upstream and downstream about each differentially expressed miRNA were listed in table. Take hsa-miR-21 for an example. Hsa-miR-21 is a well-studied oncomiR that is hyperexpressed in many cancers and may be a useful biomarker for cancer diagnosis (Banno et al., 2013).

Hsa-miR-21 is regulated by PTEN and targets at PTEN, ERBB2, TGFBR2, MSH2 and MSH6 in differentially expressed network. In related network, four more genes regulate hsa-miR-21. They are EGFR, NFKB1, REL and RELA. Hsa-miR-21 targets at 10 more genes (CDC25A, CDKN1A, E2F1, E2F2, EGFR, NCOA3, PDCD4, SERPINB5, TGIF1 and TIMP3) in related network. EGFR and hsa-miR-21 form FBLs in related network. E2F1 is a predicted TF in related network, which means hsa-miR-21 may influence more miRNAs by impacting on E2F1. In global network, hsa-miR-21 has relations with more TFs and targets. It has abundant adjacent nodes, which suggests the hsa-miR-21 is crucial in EC.

\section{Feature of pathways about predicted TFs in EC}

Then the predicted TFs which were proposed by using P-match method were focused on. Some predicted TFs interact with no miRNA in differentially expressed network and related network, which suggests that the TFs may have little influence. The predicted TFs that have upstream and downstream in differentially expressed network and related network were focused on. Take E2F1 for an example and analyze the pathway.

E2F1 regulates 5 miRNAs in differentially expressed network and 7 miRNAs in related network. It is targeted by 4 miRNAs in differentially expressed network and 5 miRNAs in related network. E2F1 is not a differentially expressed TF in EC, but it is suspicious to cause similar changes with differentially expressed TFs so that the study of predicted TFs has the enlightenment significance. HsamiR-106a and E2F1, hsa-miR-20a and E2F1 form FBLs in differentially expressed network. Hsa-miR-106b and E2F1 form FBLs in related network additionally. 


\section{Discussion}

Some special genes, miRNAs and the interactions in EC are brought to the forefront. For example, TWIST1, a helix-loop-helix transcription regulator, is known to induce epithelial-mesenchymal transition and promote tumor metastasis (Dong et al., 2014). Besides, TWIST1 also plays a part in cervical cancer (CC) which is another gynecologic cancer. It likewise regulates hsa-miR-214 and hsa-miR-214 targets at PTEN (Wang et al., 2014). There may be some similarities between EC and CC in molecular level. The predicted TFs in this study may enlighten the research of EC. To get further knowledge of genes and miRNAs in pathways relies on much more experiments. It will help much to discover the pathogenesis of EC based on the network in this study.

In conclusion, this study systematically concludes the relation of genes and miRNAs that differentially expressed in EC. Three regulatory networks which are differentially expressed network, related network and global network are constructed. The differentially expressed network is emphasized to analyze the regulation mechanism. Then upstream and downstream of differentially expressed genes, differentially expressed miRNAs and predicted TFs are extracted. Some remarkable genes and miRNAs are highlighted and their influences on other elements as well as the EC are discussed. Some pathways are proposed logically but remain to be validated by scientific experiments. This study contributes to the research of EC and provides a new vision for further research..

\section{Acknowledgements}

This work was supported by the grants from National Natural Science Foundation of China (No.60973091) and Science and Technology Development Plan of Jilin Province (No.20130101166JC).

\section{References}

Bae J, Won M, Kim DY, et al (2012). Identification of differentially expressed microRNAs in endometrial cancer cells after progesterone treatment. Int J Gynecol Cancer, 22, 561-5.

Banno K, Yanokura M, Kisu I, et al (2013). MicroRNAs in endometrial cancer. Int J Clin Oncol, 18, 186-92.

Baskerville S, Bartel DP (2005). Microarray profiling of microRNAs reveals frequent coexpression with neighboring miRNAs and host genes. RNA, 11, 241-7.

Bell DW (2014). Novel genetic targets in endometrial cancer. Expert Opin Ther Targets, 18, 725-30.

Chekmenev DS, Haid C, Kel AE (2005). P-Match: transcription factor binding site search by combining patterns and weight matrices. Nucleic Acids Res, 33, 432-7.

Cheung LWT (2012). High Frequency of PIK3R1 and PIK3R2 mutations in endometrial cancer elucidates a novel mechanism for regulation of PTENprotein stability. Cancer Discov, 2, 750-1.

Choi CH, Park YA, Choi JJ, et al (2012). Angiotensin II type I receptor and miR-155 in endometrial cancers: Synergistic antiproliferative effects of anti-miR-155 and losartan on endometrial cancer cells. Gynecol Oncol, 126, 124-31.

Cohn DE, Fabbri M, Valeri N, et al (2010). Comprehensive miRNA profiling of surgically staged endometrial cancer. Am J Obstet Gynecol, 202, 656.

Dong PX, Kaneuchi M, Watari H, Sudo S, Sakuragi N (2014). MicroRNA-106b modulates epithelial-mesenchymal transition by targeting TWIST1 in invasive endometrial cancer cell lines. Mol Carcinog, 53, 349-59.

Fujita PA, Rhead B, Zweig AS, et al (2011). The UCSC Genome Browser database: update 2011. Nucleic Acids Res, 39, 876-82.

Hobert O (2008). Gene regulation by transcription factors and microRNAs. Science, 319, 1785-6.

Hsu SD, Tseng YT, Shrestha S, et al (2014). miRTarBase update 2014: an information resource for experimentally validated miRNA-target interactions. Nucleic Acids Res, 42, 78-85.

Janiec-Jankowska A, Konopka B, Goluda C, Najmola U (2010). TP53 mutations in endometrial cancers relation to PTEN gene defects. Int J Gynecol Cancer, 20, 196-202.

Jiang QH, Wang YD, Hao YY, et al (2009). miR2Disease: a manually curated database for microRNA deregulation in human disease. Nucleic Acids Res, 37, 98-104.

Kawaguchi M, Banno K, Yanokura M, et al (2009). Analysis of candidate target genes for mononucleotide repeat mutation in microsatellite instability-high (MSI-H) endometrial cancer. Int J Oncol, 35, 977-82.

Kozomara A, Griffiths-Jones S (2011). miRBase: integrating microRNA annotation and deep-sequencing data. Nucleic Acids Res, 39, 152-7.

Krakstad C, Birkeland E, Seidel D, et al (2012). High-throughput mutation profiling of pri-mary and metastatic endometrial cancers identifies KRAS, FGFR2 and PIK3CA to Be Frequently Mutated. PLoS One, 7, 52795.

Li B, Chen H, Shang LX, Gao W (2014). MicroRNA-543 suppresses endometrial cancer on-cogenicity via targeting FAK and TWIST1 expression. Arch Gynecol Obstet, 290, 533-41.

Li BL, Lu C, Lu W, et al (2013). miR-130b is an EMTrelated microRNA that targets DIC-ER1 for aggression in endometrial cancer. Med Oncol, 30, 484.

Li BL, Lu W, Lu C, et al (2013). CpG island hypermethylationassociated silencing of microRNAs promotes human endometrial cancer. Cancer Cell Int, 13, 44.

Li J, Xu ZW, Wang KH, et al (2013). Networks of microRNAs and genes in retinoblastomas. Asian Pac J Cancer Prev, 14, 6631-6.

Lv SQ, Kim YH, Giulio F, et al (2012). Genetic Alterations in MicroRNAs in medulloblastomas. Brain Pathol, 22, 230-9.

Mackay HJ, Eisenhauer EA, Kamel-Reid S, et al (2014). Molecular determinants of outcome with mammalian target of rapamycin inhibition in endometrial cancer. Cancer, 120, 603-10.

Myatt SS, Wang J, Monteiro LJ, et al (2010). Definition of microRNAs that repress expres-sion of the tumor suppressor gene FOXO1 in endometrial cancer. Cancer Res, 70, 367-77.

Papadopoulos GL, Reczko M, Simossis VA, Sethupathy P, Hatzigeorgiou AG (2009). The database of experimentally supported targets: a functional update of TarBase. Nucleic Acids Res, 37, 155-8.

Price JC, Pollock LM, Rudd ML, et al (2013). Sequencing of candidate chromosome insta-bility genes in endometrial cancers cancers reveals somatic mutations in ESCO1, CHTF18, and MRE11A. PLoS One, 8, 63313.

Qin XY, Yan L, Zhao XB, Li CY, Fu YB (2012). microRNA-21 overexpression contributes to cell proliferation by targeting PTEN in endometrioid endometrial cancer. Oncol Lett, 4, 1290-6. 
Ramon LA, Braza-Boils A, Gilabert J, et al (2012). microRNAs related to angiogenesis are dysregulated in endometrioid endometrial cancer. Hum Reprod, 27, 3036-45.

Rodriguez A, Griffiths-Jones S, Ashurst JL, Bradley A (2004). Identification of mammalian microRNA host genes and transcription units. Genome Res, 14, 1902-10.

Samulak D, Grosman-Dziewiszek P, Michalska MM, et al (2014). Evaluation of expression of the PTEN gene, oestrogen and progesterone receptors as diagnostic and predictive factors in endometrial cancer. Pathol Oncol Res, 20, 191-6.

Shang C, Lu YM, Meng LR (2012). MicroRNA-125b downregulation mediates endometrial cancer invasion by targeting ERBB2. Med Sci Monit, 18, 149-55.

Thanapprapasr D, Thanapprapasr K (2013). Molecular therapy as a future strategy in endometrial cancer. Asian Pac J Cancer Prev, 14, 3419-23.

Torres A, Torres K, Wdowiak P, Paszkowski T, Maciejewski R (2013). Selection and validation of endogenous controls for microRNA expression studies in endometrioid endometrial cancer tissues. Gynecol Oncol, 130, 588-94.

Tran DH, Satou K, Ho TB, Pham TH (2010). Computational discovery of miR-TF regulatory modules in human genome. Bioinformation, 4, 371-7.

Tsuruta T, Kozaki K, Uesugi A, et al (2011). miR-152 Is a tumor suppressor microRNA That is silenced by DNA hypermethylation in endometrial cancer. Cancer Res, $\mathbf{7 1}$, 6450-62.

Wang HY, Shen J, Jiang CP, Liu BR (2014). How to explain the contradiction of microRNA200c expression and survival in solid tumors?: a meta-analysis. Asian Pac J Cancer Prev, 15, 3687-90.

Wang J, Lu M, Qiu CX, Cui QH (2010). TransmiR: a transcription factor-microRNA regulation database. Nucleic Acids Res, 38, 119-22.

Wang N, Xu ZW, Wang KH, Zhu MH, Li Y (2014). Construction and analysis of regulatory genetic networks in cervical cancer based on involved microRNAs, target genes, transcription factors and host genes. Oncol Lett, 7, 1279-83.

Wu D, Huang HJ, He CN, Wang KY (2013). MicroRNA-199a-3p regulates endometrial cancer cell proliferation by targeting mammalian target of rapamycin (mTOR). Int J Gynecol Cancer, 23, 1191-7.

Xiao FF, Zuo ZX, Cai GS, et al (2009). miRecords: an integrated resource for microRNA-target interactions. Nucleic Acids Res, 37, 105-10.

Xu YY, Wu HJ, Ma HD, et al (2013). MicroRNA-503 suppresses proliferation and cell-cycle progression of endometrioid endometrial cancer by negatively regulating cyclin D1. Febs $J, 280,3768-79$.

Ye WW, Xue JS, Zhang Q, et al (2014). MiR-449a functions as a tumor suppressor in endometrial cancer by targeting CDC25A. Oncol Rep, 32, 1193-9.

Zhang GY, Hou XX, Li Y, Zhao M (2014). MiR-205 inhibits cell apoptosis by targeting phosphatase and tensin homolog deleted on chromosome ten in endometrial cancer ishikawa cells. BMC Cancer, 14, 440. 